\title{
DISTORTED HANKEL INTEGRAL OPERATORS
}

\author{
A.B. ALEKSANDROV AND V.V. PELLER
}

\begin{abstract}
For $\alpha, \beta>0$ and for a locally integrable function (or, more generally, a distribution) $\varphi$ on $(0, \infty)$, we study integral ooperators $\mathfrak{G}_{\varphi}^{\alpha, \beta}$ on $L^{2}\left(\mathbb{R}_{+}\right)$ defined by $\left(\mathfrak{G}_{\varphi}^{\alpha, \beta} f\right)(x)=\int_{\mathbb{R}_{+}} \varphi\left(x^{\alpha}+y^{\beta}\right) f(y) d y$. We describe the bounded and compact operators $\mathfrak{G}_{\varphi}^{\alpha, \beta}$ and operators $\mathfrak{G}_{\varphi}^{\alpha, \beta}$ of Schatten-von Neumann class $\boldsymbol{S}_{p}$. We also study continuity properties of the averaging projection $\mathcal{Q}_{\alpha, \beta}$ onto the operators of the form $\mathfrak{G}_{\varphi}^{\alpha, \beta}$. In particular, we show that if $\alpha \leq \beta$ and $\beta>1$, then $\mathfrak{G}_{\varphi}^{\alpha, \beta}$ is bounded on $\boldsymbol{S}_{p}$ if and only if $2 \beta(\beta+1)^{-1}<p<2 \beta(\beta-1)^{-1}$.
\end{abstract}

\section{Introduction}

We are going to study a class of integral operators on $L^{2}\left(\mathbb{R}_{+}\right)$that generalizes Hankel integral operators.

For a function $\varphi$ in $L^{1}\left(\mathbb{R}_{+}\right)$, the integral Hankel operator $\boldsymbol{\Gamma}_{\varphi}$ is defined on $L^{2}\left(\mathbb{R}_{+}\right)$ by

$$
\left(\boldsymbol{\Gamma}_{\varphi} f\right)(x)=\int_{\mathbb{R}_{+}} \varphi(x+y) f(y) d y .
$$

It is easy to see that such operators are bounded on $L^{2}\left(\mathbb{R}_{+}\right)$. Operators $\boldsymbol{\Gamma}_{\varphi}$ can be bounded in a much more general case. For $\boldsymbol{\Gamma}_{\varphi}$ to be bounded, $\varphi$ does not have to be a function, it can be a distribution. Bounded Hankel operators $\boldsymbol{\Gamma}_{\varphi}$ are unitarily equivalent to Hankel operators on $\ell^{2}$, i.e., operators with Hankel matrices of the form $\left\{\alpha_{j+k}\right\}_{j, k \geq 0}$. These facts can be found in [Pel4], Ch. $1, \S 8$.

In this paper for $\alpha, \beta>0$, we study the integral operators $\mathfrak{G}_{\varphi}^{\alpha, \beta}$ on $L^{2}\left(\mathbb{R}_{+}\right)$ defined by

$$
\left(\mathfrak{G}_{\varphi}^{\alpha, \beta} f\right)(x)=\int_{\mathbb{R}_{+}} \varphi\left(x^{\alpha}+y^{\beta}\right) f(y) d y .
$$

Clearly, if $\varphi$ is a locally integrable function on $\mathbb{R}_{+}=(0, \infty)$, the right-hand side of (1.1) is well defined for smooth functions $f$ with compact support in $(0, \infty)$. The integral on the right-hand side of (1.1) makes sense for distributions $\varphi$ on $(0, \infty)$ and infinitely differentiable functions $\varphi$ with compact support in $\mathbb{R}_{+}$. We say that for a

The first author is partially supported by Grant 02-01-00267 of Russian Foundation of Fundamental Studies and by Grant 326.53 of Integration. The second author is partially supported by NSF grant DMS 0200712. 
distribution $\varphi$, the operator $\mathfrak{G}_{\varphi}^{\alpha, \beta}$ is bounded on $L^{2}\left(\mathbb{R}_{+}\right)$if it extends by continuity to $L^{2}\left(\mathbb{R}_{+}\right)$. Integral operators $\mathfrak{G}_{\varphi}^{\alpha, \beta}$ are called distorted Hankel operators. We are going to study boundedness, compactness and Schatten-von Neumann properties of distorted Hankel operators.

Obviously, for $\alpha=\beta=1$, the operator $\mathfrak{G}_{\varphi}^{\alpha, \beta}$ coincides with the integral Hankel operator $\boldsymbol{\Gamma}_{\varphi}$. On the other hand, the limit case of the operators $\mathfrak{G}_{\varphi}^{\alpha, \alpha}$ as $\alpha \rightarrow \infty$ are the integral operators $Q_{\varphi}$ on $L^{2}\left(\mathbb{R}_{+}\right)$defined by

$$
\left(Q_{\varphi} f\right)(x)=\int_{\mathbb{R}_{+}} \varphi(\max \{x, y\}) f(y) d y
$$

We refer the reader to AJPR where the operators $Q_{\varphi}$ are studied in detail. Note, however, that properties of the operators $Q_{\varphi}$ are quite different from properties of the operators $\mathfrak{G}_{\varphi}^{\alpha, \beta}$.

In $\S 2$ we collect necessary information on Schatten-von Neumann classes, weighted integral Hankel operators, and discuss properties of the averaging projection and weighted projections onto the integral Hankel operators. We state in $\S 2$ known results (Theorems A-D) that will be used in Sections 4 and 5 .

In $\S 3$ we show that Theorem B, that describes the weighted integral Hankel operators $\boldsymbol{\Gamma}_{\varphi}^{\alpha, \beta}$ of class $\boldsymbol{S}_{p}$, does not extend to $\alpha$ and $\beta$ not satisfying the hypotheses of Theorem B.

In $\S 4$ we reduce the study of the operators $\mathfrak{G}_{\varphi}^{\alpha, \beta}$ to the study of weighted integral Hankel operators (see the definition in $\S 2$ ). We describe the operators $\mathfrak{G}_{\varphi}^{\alpha, \beta}$ that belong to the Schatten-von Neumann class $\boldsymbol{S}_{p}$ under a certain condition on $\alpha, \beta$, and $\mathcal{P}$.

In $\S 5$ we introduce the averaging projection $\mathcal{Q}_{\alpha, \beta}$ onto the subspace of operators of the form $\mathfrak{G}_{\varphi}^{\alpha, \beta}$ and we study their metric properties. In particular, we show that if $\alpha \leq \beta, \beta>1$, and

$$
\frac{2 \beta}{\beta+1}<p<\frac{2 \beta}{\beta-1}
$$

then $\mathcal{Q}_{\alpha, \beta}$ is bounded on $\boldsymbol{S}_{p}$. Moreover, this result is sharp. It is interesting to observe that both $\frac{2 \beta}{\beta+1}$ and $\frac{2 \beta}{\beta-1}$ go to 2 as $\beta$ tends to $\infty$. However, in the limiting case the averaging projection on the subspace of the operators of the from $Q_{\varphi}$ is bounded for all $p \in(1, \infty)$, see AJPR.

It is also interesting that, unlike in the case of Hankel integral operators, for $\alpha, \beta \in(0,1)$, the averaging projection $\mathcal{Q}_{\alpha, \beta}$ is bounded on $\boldsymbol{S}_{1}$, and on the space of bounded and compact operators. 


\section{Preliminaries}

Schatten-von Neumann classes. Recall that for a bounded operator $T$ on a Hilbert space $\mathcal{H}$ the singular values $s_{j}(T), j \geq 0$, are defined by

$$
s_{j}(T)=\inf \{\|T-K\|: K \in \mathcal{B}(\mathcal{H}), \operatorname{rank} K \leq j\} .
$$

Here $\mathcal{B}(\mathcal{H})$ denotes the space of bounded linear operators on $\mathcal{H}$.

The Schatten-von Neumann class $\boldsymbol{S}_{p}=\boldsymbol{S}_{p}(\mathcal{H}), 0<p<\infty$, consists of the operators $T$ on $\mathcal{H}$ such that

$$
\|T\|_{\boldsymbol{S}_{p}}=\left(\sum_{j \geq 0}\left(s_{j}(T)\right)^{p}\right)^{1 / p}<\infty .
$$

We denote by $\mathcal{C}(\mathcal{H})$ the space of compact operators on $\mathcal{H}$.

If $1 \leq p<\infty$, then $\|\cdot\|_{\boldsymbol{S}_{p}}$ is a norm, which makes $\boldsymbol{S}_{p}$ a Banach space. For $p<1,\|\cdot\|_{\boldsymbol{S}_{p}}$ does not satisfy the triangle inequality, it is a quasinorm (i.e., $\left\|T_{1}+T_{2}\right\|_{\boldsymbol{S}_{p}} \leq \operatorname{const}\left(\left\|T_{1}\right\|_{\boldsymbol{S}_{p}}+\left\|T_{2}\right\|_{\boldsymbol{S}_{p}}\right)$ for $\left.T_{1}, T_{2} \in \boldsymbol{S}_{p}\right)$, which makes $\boldsymbol{S}_{p}$ a quasiBanach space.

The linear functional trace is defined on $\boldsymbol{S}_{1}$ by

$$
\operatorname{trace} T=\sum_{j \geq 0}\left(T e_{j}, e_{j}\right), \quad T \in \boldsymbol{S}_{1},
$$

where $\left\{e_{j}\right\}_{j \geq 0}$ is an orthonormal basis in $\mathcal{H}$. Moreover, the right-hand side does not depend on the choice of the basis.

If $1<p<\infty$, the dual space $\boldsymbol{S}_{p}^{*}$ can be identified with $\boldsymbol{S}_{p^{\prime}}$ with respect to the pairing

$$
\langle T, R\rangle=\operatorname{trace} T R^{*}, \quad T \in \boldsymbol{S}_{p}, \quad R \in \boldsymbol{S}_{p^{\prime}} .
$$

Here $p^{\prime}=p /(p-1)$ is the dual exponent. With respect to the same pairing (2.1) one can identify $\boldsymbol{S}_{1}^{*}$ with $\mathcal{B}(\mathcal{H})$ and $(\mathcal{C}(\mathcal{H}))^{*}$ with $\boldsymbol{S}_{1}$.

In the case $\mathcal{H}=L^{2}(\mathcal{X}, \mu)$ and $\mu$ is a $\sigma$-finite measure, the space $\boldsymbol{S}_{2}$ coincides with the set of integral operators $\mathfrak{I}_{k}$,

$$
\left(\mathfrak{I}_{k} f\right)(x)=\int_{\mathcal{X}} k(x, y) f(y) d \mu(y), \quad f \in L^{2}(\mathcal{X}, \mu), \quad x, y \in \mathcal{X}
$$

with $k \in L^{2}(\mathcal{X} \times \mathcal{X}, \mu \otimes \mu)$ and

$$
\left\|\mathfrak{I}_{k}\right\|_{S_{2}}=\left(\iint_{\mathcal{X} \times \mathcal{X}}|k(x, y)|^{2} d \mu(x) d \mu(y)\right)^{1 / 2} .
$$


Moreover, if $T$ and $R$ are integral operators in $\boldsymbol{S}_{2}$ with kernel functions $k$ and $\xi$, then

$$
\operatorname{trace} T R^{*}=\iint_{\mathcal{X} \times \mathcal{X}} k(x, y) \overline{\xi(x, y)} d \mu(x) d \mu(y) .
$$

We refer the reader to [GK] and BS for basic facts about Schatten-von Neumann classes.

Besov classes. We consider here spaces $\mathfrak{B}_{p}^{s}$ of distributions on $\mathbb{R}_{+}=(0, \infty)$. The space $\mathfrak{B}_{p}^{s}$ can be identified with the restrictions of the Fourier transforms of functions in the Besov classes $B_{p}^{s}(\mathbb{R})$ to $\mathbb{R}_{+}$.

Let $v$ be a $C^{\infty}$ function on $\mathbb{R}$ such that

$$
\operatorname{supp} v=\left[\frac{1}{2}, 2\right] \text { and } \sum_{j=-\infty}^{\infty} v\left(\frac{x}{2^{j}}\right)=1, \quad x>0 .
$$

Put

$$
v_{j}(x)=v\left(\frac{x}{2^{j}}\right), \quad x>0 .
$$

For $0<p \leq \infty$ and $s \in \mathbb{R}$, we define the space $\mathfrak{B}_{p}^{s}$ as the space of distributions $\varphi$ on $\mathbb{R}_{+}$such that

$$
\|\varphi\|_{\mathfrak{B}_{p}^{s}} \stackrel{\text { def }}{=}\left(\sum_{j=-\infty}^{\infty}\left(2^{j s}\left\|\mathcal{F}\left(v_{j} \varphi\right)\right\|_{L^{p}}\right)^{p}\right)^{1 / p}<\infty, \quad p<\infty,
$$

and

$$
\|\varphi\|_{\mathfrak{B}_{\infty}^{s}} \stackrel{\text { def }}{=} \sup _{-\infty<j<\infty} 2^{j s}\left\|\mathcal{F}\left(v_{j} \varphi\right)\right\|_{L^{\infty}}<\infty .
$$

Here $\mathcal{F}$ denotes the Fourier transform.

If $p \geq 1, \mathfrak{B}_{p}^{s}$ is a Banach space with norm $\|\cdot\|_{\mathfrak{B}_{p}^{s}}$. If $p<1, \mathfrak{B}_{p}^{s}$ is a quasi-Banach space with quasinorm $\|\cdot\|_{\mathfrak{B}_{p}^{s}}$, i.e., $\left\|\varphi_{1}+\varphi_{2}\right\|_{\mathfrak{B}_{p}^{s}} \leq \operatorname{const}\left(\left\|\varphi_{1}\right\|_{\mathfrak{B}_{p}^{s}}+\left\|\varphi_{2}\right\|_{\mathfrak{B}_{p}^{s}}\right)$.

We also define the space $\mathfrak{b}_{\infty}^{s}, s \in \mathbb{R}$, as the closed subspace of $\mathfrak{B}_{\infty}^{s}$, which consists of distributions $\varphi \in \mathfrak{B}_{\infty}^{s}$ such that

$$
\lim _{|j| \rightarrow \infty} 2^{j s}\left\|\mathcal{F}\left(v_{j} \varphi\right)\right\|_{L^{\infty}}=0 .
$$

If $\varphi$ is a distribution on $\mathbb{R}_{+}$and $\psi(x)=x^{\sigma} \varphi(x)$ (this equality has to be understood in the distributional sense), then it is easy to see that $\varphi \in \mathfrak{B}_{\infty}^{s}$ if and only if $\psi \in \mathfrak{B}_{\infty}^{s-\sigma}$.

If $1 \leq p<\infty$ and $s_{1}, s_{2} \in \mathbb{R}$, one can identify the dual space $\left(\mathfrak{B}_{p}^{s_{1}}\right)^{*}$ with the space $\mathfrak{B}_{p^{\prime}}^{s_{2}}$ with respect to the pairing

$$
\langle\varphi, \psi\rangle=\int_{0}^{\infty} t^{s_{1}+s_{1}} \varphi(t) \overline{\psi(t)} d t, \quad \varphi \in \mathfrak{B}_{p}^{s_{1}}, \quad \psi \in \mathfrak{B}_{p^{\prime}}^{s_{2}}
$$


Note that the integral on the right-hand side makes sense for compactly supported $C^{\infty}$ functions $\varphi$ can be understood as the value of the distribution $\psi$ at the function $t \mapsto t^{s_{1}+s_{1}} \varphi(t)$. The linear functional $\varphi \mapsto\langle\varphi, \psi\rangle$ extends by continuity to the whole space $\mathfrak{B}_{p}^{s_{1}}$. As usual, $\frac{1}{p}+\frac{1}{p^{\prime}}=1$.

The dual space $\mathfrak{b}_{\infty}^{s_{1}}$ can be identified with the space $\mathfrak{B}_{1}^{s_{2}}$ with respect to the same pairing (2.3).

Remark. The Besov spaces $B_{p}^{s}(\mathbb{R})$ of functions on $\mathbb{R}$ can be defined as the space of tempered distributions $f$ on $\mathbb{R}$ such that

$$
\sum_{j=-\infty}^{\infty}\left(2^{s j}\left\|f * \xi_{j}\right\|_{L^{p}}\right)^{p}+\sum_{j=-\infty}^{\infty}\left(2^{s j}\left\|f * \eta_{j}\right\|_{L^{p}}\right)^{p}<\infty
$$

where $\xi_{j}$ and $\eta_{j}$ are functions in on $\mathbb{R}$ such that

$$
\mathcal{F} \xi_{j}=v_{j} \quad \text { and } \quad\left(\mathcal{F} \eta_{j}\right)(x)=v_{j}(-x), \quad x \in \mathbb{R} .
$$

and the $v_{j}$ are defined by (2.2). This this space contains all polynomials. It is possible to define the space $B_{p}^{s}(\mathbb{R})$ modulo the polynomials of degree at most $s-1 / p$, in which case only polynomials of degree at most $s-1 / p$ can belong to this space. In both cases

$$
\mathfrak{B}_{p}^{s}=\left\{\mathcal{F} f \mid(0, \infty): f \in B_{p}^{s}(\mathbb{R})\right\} .
$$

The subspace $\left(B_{p}^{s}(\mathbb{R})\right)_{+}$of $B_{p}^{s}(\mathbb{R})$ is defined by

$$
\left(B_{p}^{s}(\mathbb{R})\right)_{+}=\left\{f \in B_{p}^{s}(\mathbb{R}): \operatorname{supp} \mathcal{F} f \subset[0,+\infty)\right\} .
$$

Functions in $\left(B_{p}^{s}(\mathbb{R})\right)_{+}$can be extended analytically in a natural way to the upper half-plane. Clearly,

$$
\left\{(\mathcal{F} f) \mid(0, \infty): f \in\left(B_{p}^{s}(\mathbb{R})\right)_{+}\right\}=\mathfrak{B}_{p}^{s} .
$$

We refer the reader to $\mathrm{Pee}]$ for more information about Besov classes.

Weighted integral Hankel operators. For a locally integrable function $\varphi$ on $\mathbb{R}_{+}$the weighted integral Hankel operator $\boldsymbol{\Gamma}_{\varphi}^{\alpha, \beta}$ is defined by

$$
\left(\boldsymbol{\Gamma}_{\varphi}^{\alpha, \beta} f\right)(x)=\int_{0}^{\infty} x^{\alpha} y^{\beta} \varphi(x+y) f(y) d y
$$

for smooth functions $f$ with compact support in $\mathbb{R}_{+}$. Again, the definition makes sense for distributions $\varphi$ on $\mathbb{R}_{+}$. The operators $\boldsymbol{\Gamma}_{\varphi}^{\alpha, \beta}$ are analogs of weighted Hankel matrices $\Gamma_{\psi}^{\alpha, \beta}=\left\{(1+j)^{\alpha}(1+k)^{\beta} \hat{\psi}(j+k)\right\}_{j, k \geq 0}$, where $\psi$ is a function analytic in the unit disk.

For $\alpha=\beta=0$, the operator $\boldsymbol{\Gamma}_{\varphi}^{0,0}=\boldsymbol{\Gamma}_{\varphi}$ is the integral Hankel operator defined in the introduction. 
We need the following results.

Theorem A. Suppose that $\alpha>0$ and $\beta>0$. Then $\Gamma_{\varphi}^{\alpha, \beta}$ is bounded on $L^{2}\left(\mathbb{R}_{+}\right)$ if and only if $\varphi \in \mathfrak{B}_{\infty}^{\alpha+\beta}$ and $\boldsymbol{\Gamma}_{\varphi}^{\alpha, \beta}$ is compact if and only if $\varphi \in \mathfrak{b}_{\infty}^{\alpha+\beta}$.

We refer the reader to $\mathrm{Pel} 2$ for the corresponding result for weighted Hankel matrices and to [DP] for more general results than Theorem A.

Theorem B. Let $0<p<\infty$. Suppose that $\min \{\alpha, \beta\}>\max \left\{-\frac{1}{2},-\frac{1}{p}\right\}$. Then $\boldsymbol{\Gamma}_{\varphi}^{\alpha, \beta} \in \boldsymbol{S}_{p}$ if and only if $\varphi \in \mathfrak{B}_{p}^{1 / p+\alpha+\beta}$.

For $p \geq 1$ the description of the weighted Hankel matrices $\Gamma_{\psi}^{\alpha, \beta}$ of class $\boldsymbol{S}_{p}$ was obtained in [Pel1] for $\alpha=\beta=0$ and in $\mathbb{P e l 2}$ in the case $\min \{\alpha, \beta\}>\max \left\{-\frac{1}{2},-\frac{1}{p}\right\}$. For integral Hankel operators and $p \geq 1$ see [CR, $[\mathbb{R}]$, and [DP]. In the case $p<1$ we refer the reader to [Pel1] for weighted Hankel matrices and to [S] for the weighted integral Hankel operators. See also [Pel4], Ch. 6.

Averaging projection onto the integral Hankel operators. Consider the orthogonal projection $\mathcal{P}$ on the Hilbert-Schmidt class $\boldsymbol{S}_{2}$ onto the subspace of Hankel integral operators. Clearly, if $k \in L^{2}\left(\mathbb{R}_{+} \times \mathbb{R}_{+}\right)$, then

$$
\mathcal{P} \mathfrak{I}_{k}=\Gamma_{\varphi},
$$

where

$$
\varphi(x)=\frac{1}{x} \int_{0}^{x} k(t, x-t) d t .
$$

For $2<p<\infty$, the averaging projection $\mathcal{P}$ can be defined on the dense subset $\boldsymbol{S}_{p} \cap \boldsymbol{S}_{2}$ of of $\boldsymbol{S}_{p}$. As usual, we say that $\mathcal{P}$ is bounded on $\boldsymbol{S}_{p}$ if it extends by continuity to a bounded operator on $\boldsymbol{S}_{p}$.

Theorem C. Let $1<p<\infty$. Then the averaging projection $\mathcal{P}$ is bounded on $S_{p}$.

The same result for the averaging projection onto the space of Hankel matrices was obtained in Pel1], see also [Pel4]. The same proof works for the averaging projection onto the set of integral Hankel operators.

Note that $\mathcal{P}$ is unbounded on $\boldsymbol{S}_{1}$ and on the spaces of bounded and compact operators (see [Pel1], [Pel4]). However, there are bounded projections on $\boldsymbol{S}_{1}$ onto the subspace of integral Hankel operators. Indeed, for $\alpha, \beta>0$ we define the weighted averaging projection $\mathcal{P}_{\alpha, \beta}$ by

$$
\mathcal{P}_{\alpha, \beta} \mathfrak{I}_{k}=\Gamma_{\varphi},
$$


where

$$
\varphi(x)=\frac{\int_{0}^{x} t^{\alpha}(x-t)^{\beta} k(t, x-t) d t}{\int_{0}^{x} t^{\alpha}(x-t)^{\beta} d t} .
$$

Theorem D. Let $\alpha, \beta>0$. Then $\mathcal{P}_{\alpha, \beta}$ is a bounded operator on $\boldsymbol{S}_{1}$.

The corresponding result for weighted averaging projection on the subspace of Hankel matrices was found by the first author, see ([Pel2 and [Pel4] $)$. The same proof works in the case of integral operators.

\section{Theorem $B$ is sharp}

When we study properties of the averaging projection $\mathcal{Q}_{\alpha, \beta}$ onto the space of operators of the form $\mathfrak{G}_{\varphi}^{\alpha, \beta}$, we will need the fact that Theorem $\mathrm{B}$ cannot be extended to other values of $\alpha$ and $\beta$.

Theorem 3.1. Let $\alpha, \beta \in \mathbb{R}$ and $0<p<\infty$. Suppose that $\min \{\alpha, \beta\} \leq$ $\max \left\{-\frac{1}{2},-\frac{1}{p}\right\}$. Then there are functions $\psi \in \mathfrak{B}_{p}^{1 / p+\alpha+\beta}$ such that $\boldsymbol{\Gamma}_{\psi}^{\alpha, \beta} \notin \boldsymbol{S}_{p}$.

Proof. Consider first the case $p \geq 2$. For a positive integer $n$ we define the function $\varphi_{n}$ on $\mathbb{R}_{+}$by

$$
\varphi_{n}(x)= \begin{cases}1, & x \in\left(1,1+\frac{2}{n}\right) \\ 0, & \text { otherwise }\end{cases}
$$

It follows easily from the definition of $\mathfrak{B}_{p}^{1 / p+\alpha+\beta}$ that

$$
\left\|\varphi_{n}\right\|_{\mathfrak{B}_{p}^{1 / p+\alpha+\beta}} \leq \mathrm{const}\left\|\mathcal{F} \varphi_{n}\right\|_{L^{p}} \leq \mathrm{const} \cdot n^{-1 / p^{\prime}}
$$

Let us now estimate from below $\left\|\boldsymbol{\Gamma}_{\varphi_{n}}^{\alpha, \beta}\right\|_{\boldsymbol{S}_{p}}$. Define the function $k$ on $\mathbb{R}_{+}^{2}$ by

$$
k(x, y) \stackrel{\text { def }}{=} \begin{cases}x^{\alpha} y^{\beta}, & (x . y) \in \bigcup_{k=0}^{n} \Delta_{j}, \\ 0, & (x, y) \notin \bigcup_{k=0}^{n} \Delta_{j}\end{cases}
$$

where

$$
\Delta_{j}=\left(\frac{j}{n}, \frac{j+1}{n}\right) \times\left(\frac{n-j}{n}, \frac{n-j+1}{n}\right), \quad 0 \leq j \leq n
$$


Since the squares $\Delta_{j}$ have disjoint projections onto the coordinate axes, it is easy to see that

$$
\left\|\boldsymbol{\Gamma}_{\varphi_{n}}^{\alpha, \beta}\right\|_{\boldsymbol{S}_{p}} \geq\left\|\mathfrak{I}_{k}\right\|_{\boldsymbol{S}_{p}}=\left(\sum_{j=0}^{n}\left\|\mathfrak{I}_{k_{j}}\right\|_{\boldsymbol{S}_{p}}^{p}\right)^{1 / p}
$$

where $k_{j}=k \chi_{\Delta_{j}}$ and $\chi_{\Delta_{j}}$ is the characteristic function of $\Delta_{j}$. Clearly, $\mathfrak{I}_{k_{j}}$ is a rank one operator and

$$
\left\|\Im_{k_{j}}\right\|_{\boldsymbol{S}_{p}} \geq \frac{j^{\alpha}(n-j)^{\beta}}{n^{\alpha+\beta+1}}
$$

Without loss of generality, we may assume $\alpha \leq \beta$. It is easy to verify that

$$
\begin{aligned}
\left\|\boldsymbol{\Gamma}_{\varphi_{n}}^{\alpha, \beta}\right\|_{\boldsymbol{S}_{p}} & \geq\left(\sum_{j=0}^{n} \frac{j^{p \alpha}(n-j)^{p \beta}}{n^{p(\alpha+\beta+1)}}\right)^{1 / p} \\
& \geq \operatorname{const} \begin{cases}n^{-\alpha-1}, & \alpha<-1 / p \\
n^{-\alpha-1}(\log (1+n))^{1 / p}, & \alpha=-1 / p .\end{cases}
\end{aligned}
$$

Clearly,

$$
\frac{\left\|\Gamma_{\varphi_{n}}^{\alpha, \beta}\right\|_{\boldsymbol{S}_{p}}}{\left\|\varphi_{n}\right\|_{\mathfrak{B}_{p}^{1 / p+\alpha+\beta}}} \rightarrow \infty \quad \text { as } \quad n \rightarrow \infty
$$

which completes the proof in the case $p \geq 2$.

Suppose now that $p<2$. Again, we assume that $\alpha \leq \beta$. We prove that if $\alpha \leq-\frac{1}{2}$, the condition $\psi \in \mathfrak{B}_{p}^{1 / p+\alpha+\beta}$ does not even imply that $\boldsymbol{\Gamma}_{\psi}^{\alpha, \beta} \in \boldsymbol{S}_{2}$. Let $\psi$ be a nonzero smooth function with support in $[1,2]$. Clearly, $\mathcal{F} \psi \in L^{p}$, and so $\psi \in \mathfrak{B}_{p}^{1 / p+\alpha+\beta}$. On the other hand,

$$
\left\|\boldsymbol{\Gamma}_{\psi}^{\alpha, \beta}\right\|_{\boldsymbol{S}_{2}}^{2}=\int_{1}^{2}|\varphi(t)|^{2} \int_{0}^{t} x^{2 \alpha}(t-x)^{2 \beta} d x d t=\infty
$$

since, clearly,

$$
\int_{0}^{t} x^{2 \alpha}(t-x)^{2 \beta} d x=\infty
$$

\section{Boundedness, Compactness, and Schatten-von Neumann Properties}

Let $k$ be a function on $\mathbb{R}_{+}^{2}$ such that the integral operator on $L^{2}\left(\mathbb{R}_{+}\right)$with kernel function $k$ is bounded on $L^{2}\left(\mathbb{R}_{+}\right)$. As in $\S 2$, denote this integral operator by $\mathfrak{I}_{k}$ :

$$
\left(\mathfrak{I}_{k} f\right)(x)=\int_{\mathbb{R}_{+}} k(x, y) f(y) d y .
$$


We say that $k \in \boldsymbol{S}_{p}\left(\mathbb{R}_{+}^{2}\right)$ if the operator $\mathfrak{I}_{k}$ belongs to the Schatten-von Neumann class $\boldsymbol{S}_{p}$ and we write $\|k\|_{\boldsymbol{S}_{p}} \stackrel{\text { def }}{=}\left\|\mathfrak{I}_{k}\right\|_{\boldsymbol{S}_{p}}$. Let now $\alpha$ and $\beta$ be nonzero real numbers. We put

$$
k_{\alpha, \beta}(x, y) \stackrel{\text { def }}{=} x^{\frac{1}{2 \alpha}-\frac{1}{2}} y^{\frac{1}{2 \beta}-\frac{1}{2}} k\left(x^{\frac{1}{\alpha}}, y^{\frac{1}{\beta}}\right) .
$$

We introduce the unitary operator $U_{\alpha}$ on $L^{2}\left(\mathbb{R}_{+}\right)$defined by

$$
\left(U_{\alpha} f\right)(x)=\frac{1}{\sqrt{|\alpha|}} x^{\frac{1}{2 \alpha}-\frac{1}{2}} f\left(x^{\frac{1}{\alpha}}\right), \quad f \in L^{2}\left(\mathbb{R}_{+}\right) .
$$

It is easy to see that

$$
U_{\alpha} \Im_{k}=\frac{1}{\sqrt{|\alpha \beta|}} \mathfrak{I}_{k_{\alpha, \beta}} U_{\beta}, \quad \alpha, \beta \in \mathbb{R} \backslash\{0\},
$$

and so

$$
\left\|k_{\alpha, \beta}\right\|_{\boldsymbol{S}_{p}}=\sqrt{|\alpha \beta|} \cdot\|k\|_{\boldsymbol{S}_{p}} .
$$

Theorem 4.1. Suppose that $\alpha, \beta \in(0,1)$. Then $\mathfrak{G}_{\varphi}^{\alpha, \beta}$ is bounded if and only if $\varphi \in \mathfrak{B}_{\infty}^{\frac{1}{2 \alpha}+\frac{1}{2 \beta}-1}$. The operator $\mathfrak{G}_{\varphi}^{\alpha, \beta}$ is compact if and only if $\varphi \in \mathfrak{b}_{\infty}^{\frac{1}{2 \alpha}+\frac{1}{2 \beta}-1}$.

Proof. Suppose first that $\varphi$ is a locally integrable functin on $\mathbb{R}_{+}$. Consider the kernel function $\varkappa$ of $\mathfrak{G}^{\alpha, \beta}$ :

$$
\varkappa(x, y)=\varphi\left(x^{\alpha}+y^{\beta}\right), \quad x, y>0 .
$$

Obviously, by (4.1)

$$
\varkappa_{\alpha, \beta}(x, y)=x^{\frac{1}{2 \alpha}-\frac{1}{2}} y^{\frac{1}{2 \beta}-\frac{1}{2}} \varphi(x+y),
$$

i.e., the integral operator operator with kernel function $\varkappa_{\alpha, \beta}$ is a weighted integral Hankel operator. Hence, $\mathfrak{G}_{\varphi}^{\alpha, \beta}$ is bounded (or compact) if and only if $\boldsymbol{\Gamma}_{\varphi}^{\frac{1}{2 \alpha}-\frac{1}{2}, \frac{1}{2 \beta}-\frac{1}{2}}$ is bounded (or compact). By Theorem A (see $\S 2$ ), this is equivalent to the fact that $\varphi \in \mathfrak{B}_{\infty}^{\frac{1}{2 \alpha}+\frac{1}{2 \beta}-1}$ (or $\left.\varphi \in \mathfrak{b}_{\infty}^{\frac{1}{2 \alpha}+\frac{1}{2 \beta}-1}\right)$.

If $\varphi$ is a distribution, it is easy to verify that the formula

$$
\Gamma_{\varphi}^{\frac{1}{2 \alpha}-\frac{1}{2}, \frac{1}{2 \beta}-\frac{1}{2}}=\sqrt{\alpha \beta} U_{\alpha} \mathfrak{G}_{\varphi}^{\alpha, \beta} U_{\beta}^{*}
$$

still holds, which implies the result.

Theorem 4.2. Let $p, \alpha$, and $\beta$ be positive numbers such that

$$
\max \{\alpha, \beta\}(p-2)<p .
$$

Then $\mathfrak{G}_{\varphi}^{\alpha, \beta} \in \boldsymbol{S}_{p}$ if and only if $\varphi \in \mathfrak{B}_{p}^{\frac{1}{2 \alpha}+\frac{1}{2 \beta}+\frac{1}{p}-1}$. 
Proof. Again, apply formula (4.3). Obviously, $\alpha$ and $\beta$ satisfy the hypotheses of Theorem B. The result follows from Theorem B.

It turns out that in the case $1 \leq p<\infty$ the necessity of the condition $\varphi \in \mathfrak{B}_{p}^{\frac{1}{2 \alpha}+\frac{1}{2 \beta}+\frac{1}{p}-1}$ holds for any positive $\alpha$ and $\beta$.

Theorem 4.3. Let $1 \leq p<\infty$, and let $\alpha$ and $\beta$ be positive numbers. Suppose that $\mathfrak{G}_{\varphi}^{\alpha, \beta} \in \boldsymbol{S}_{p}$. Then $\varphi \in \mathfrak{B}_{p}^{\frac{1}{2 \alpha}+\frac{1}{2 \beta}+\frac{1}{p}-1}$.

Proof. As we have already observed, the integral operator $\mathfrak{I}_{\varkappa_{\alpha, \beta}}$ with kernel function $\varkappa_{\alpha, \beta}$ defined by (4.2) must belong to $\boldsymbol{S}_{p}$.

Suppose first that $p>1$. We apply to $\mathfrak{I}_{\varkappa_{\alpha, \beta}}$ the averaging projection $\mathcal{P}($ see $\S 2)$. It is easy to verify that

$$
\mathcal{P} \mathfrak{I}_{\varkappa_{\alpha, \beta}}=\operatorname{const} \boldsymbol{\Gamma}_{\psi}
$$

where

$$
\psi(x)=x^{\frac{1}{2 \alpha}+\frac{1}{2 \beta}-1} \varphi(x) .
$$

By Theorem C, $\boldsymbol{\Gamma}_{\psi} \in \boldsymbol{S}_{p}$. Now by Theorem B, $\psi \in \mathfrak{B}_{p}^{1 / p}$, which is equivalent to the fact that $\varphi \in \mathfrak{B}_{p}^{\frac{1}{2 \alpha}+\frac{1}{2 \beta}+\frac{1}{p}-1}$ (see Section 2).

Let now $p=1$. We apply to the operator $\mathfrak{I}_{\varkappa_{\alpha, \beta}}$ the weighted projection $\mathcal{P}_{1.1}$, which is bounded on $\boldsymbol{S}_{1}$ (see Theorem D). It is easy to verify that

$$
\mathcal{P}_{1,1} \mathfrak{I}_{\varkappa_{\alpha, \beta}}=\text { const } \boldsymbol{\Gamma}_{\psi},
$$

where $\psi$ is defined by (4.4). By Theorem $\mathrm{B}, \psi \in \mathfrak{B}_{1}^{1}$, which is equivalent to the fact that $\varphi \in \mathfrak{B}_{1}^{\frac{1}{2 \alpha}+\frac{1}{2 \beta}}($ see $\S 2)$.

\section{The Averaging Projection onto the Operators $\mathfrak{G}_{\varphi}^{\alpha, \beta}$}

In this section we study metric properties of the averaging projection on the class of operators of the form $\mathfrak{G}_{\varphi}^{\alpha, \beta}$. Consider the orthogonal projection $\mathcal{Q}_{\alpha, \beta}$ from the Hilbert-Schmidt class $\boldsymbol{S}_{2}$ onto the subspace of $\boldsymbol{S}_{2}$ of operators of the form $\mathfrak{G}_{\varphi}^{\alpha, \beta}$. Clearly, $\mathcal{Q}_{1,1}$ is just the averaging projection $\mathcal{P}$ onto the Hankel integral operators. If we identify the Hilbert-Schmidt operators on $L^{2}\left(\mathbb{R}_{+}\right)$with the space $L^{2}\left(\mathbb{R}_{+}^{2}\right)$, we find that $\mathcal{Q}_{\alpha, \beta}$ is the orthogonal projection onto the subspace of functions that are constant on the sets $\left\{(x, y) \in \mathbb{R}_{+}^{2}: x^{\alpha}+y^{\beta}=c\right\}, c>0$.

We are going to characterize those $\alpha, \beta$, and $p$, for which the projection $\mathcal{Q}_{\alpha, \beta}$ is bounded on $\boldsymbol{S}_{p}$. Clearly, $\mathcal{Q}_{\alpha, \beta} T$ is well-defined for $T \in \boldsymbol{S}_{p}$ if $p \leq 2$. If $p>2$, we say that $\mathcal{Q}_{\alpha, \beta}$ is bounded on $\boldsymbol{S}_{p}$ if it extends to a bounded operator from $\boldsymbol{S}_{p} \cap \boldsymbol{S}_{2}$. Since $\mathcal{Q}_{\alpha, \beta}$ is self-adjoint on $\boldsymbol{S}_{2}$, it follows that $\mathcal{Q}_{\alpha, \beta}$ is bounded on $\boldsymbol{S}_{p}$ if and only if it is bounded on $\boldsymbol{S}_{p^{\prime}}, 1<p<\infty$, and $\mathcal{Q}_{\alpha, \beta}$ is bounded on $\boldsymbol{S}_{1}$ if and only if it is bounded on $\mathcal{B}\left(L^{2}\left(\mathbb{R}_{+}\right)\right)$. 
Lemma 5.1. Let $k \in L^{2}\left(\mathbb{R}_{+}^{2}\right)$ and let $\alpha, \beta>0$. Put

$$
\varphi(x) \stackrel{\int_{0}^{\frac{\pi}{2}} k\left(x^{\frac{1}{\alpha}} \cos ^{\frac{2}{\alpha}} t, x^{\frac{1}{\beta}} \sin ^{\frac{2}{\beta}} t\right) \cos ^{\frac{2}{\alpha}-1} t \sin ^{\frac{2}{\beta}-1} t d t}{A(\alpha, \beta)},
$$

where

$$
A(\alpha, \beta) \stackrel{\text { def }}{=} \int_{0}^{\frac{\pi}{2}} \cos ^{\frac{2}{\alpha}-1} t \sin ^{\frac{2}{\beta}-1} t d t
$$

Then

$$
\mathcal{Q}_{\alpha, \beta} \mathfrak{I}_{k}=\mathfrak{G}_{\varphi}^{\alpha, \beta} .
$$

Proof. The result follows from the following easily verifiable formula:

$$
\iint_{\mathbb{R}_{+}^{2}} f(x, y) d x d y=\frac{2}{\alpha \beta} \int_{0}^{\infty} r^{\frac{1}{\alpha}+\frac{1}{\beta}-1} \int_{0}^{\frac{\pi}{2}} f\left(r^{\frac{1}{\alpha}} \cos ^{\frac{2}{\alpha}} t, r^{\frac{1}{\beta}} \sin ^{\frac{2}{\beta}} t\right) \cos ^{\frac{2}{\alpha}-1} t \sin ^{\frac{2}{\beta}-1} t d t d r,
$$

which holds for any nonnegative measurable function $f$.

Remark. Note that $A(\alpha, \beta)=\frac{1}{2} B(1 / \alpha, 1 / \beta)$, where $B$ is the Euler Beta function.

Theorem 5.2. Let $1 \leq p<\infty$, and let $\alpha$ and $\beta$ be positive numbers. Suppose that

$$
-p<\max \{\alpha, \beta\}(p-2)<p .
$$

Then $\mathcal{Q}_{\alpha, \beta}$ is bounded on $\boldsymbol{S}_{p}$.

Note that if $\max \{\alpha, \beta\} \leq 1$, the rightmost inequality in (5.4) holds for any $p$. If $\max \{\alpha, \beta\}>1$, then (5.4) is equivalent to the inequalities

$$
\frac{2 \max \{\alpha, \beta\}}{\max \{\alpha, \beta\}+1}<p<\frac{2 \max \{\alpha, \beta\}}{\max \{\alpha, \beta\}-1} .
$$

Clearly, $\frac{2 \max \{\alpha, \beta\}}{\max \{\alpha, \beta\}+1}$ and $\frac{2 \max \{\alpha, \beta\}}{\max \{\alpha, \beta\}-1}$ are dual exponents, i.e., the sum of their reciprocals is euqal to one.

Proof. Consider first the case $p>1$. Let $\mathcal{T}_{\alpha, \beta}$ be the operator on $\boldsymbol{S}_{p}$ defined by

$$
\mathcal{T}_{\alpha, \beta} T=\varphi, \quad \text { where } \quad \mathcal{Q}_{\alpha, \beta} T=\mathfrak{G}_{\varphi}^{\alpha, \beta},
$$

see Lemma 5.1. By Theorem 4.2, we have to show that $\mathcal{T}_{\alpha, \beta}$ is a bounded operator from $\boldsymbol{S}_{p}$ to $\mathfrak{B}_{p}^{\frac{1}{p}+\frac{1}{2 \alpha}+\frac{1}{2 \beta}-1}$. 
Consider the dual exponent $p^{\prime}=p /(p-1)$. Define the operator

$$
\mathcal{R}: \mathfrak{B}_{p^{\prime}}^{\frac{1}{p^{\prime}}+\frac{1}{2 \alpha}+\frac{1}{2 \beta}-1} \rightarrow \boldsymbol{S}_{p^{\prime}}\left(\mathbb{R}_{+}^{2}\right)
$$

by

$$
(\mathcal{R} \varphi)(x, y)=\varphi\left(x^{\alpha}+y^{\beta}\right), \quad x, y>0 .
$$

By Theorem $1.2, \mathcal{R}$ is a bounded operator.

Let $k \in \boldsymbol{S}_{1}\left(\mathbb{R}_{+}^{2}\right)$ and let

$$
\mathcal{Q}_{\alpha, \beta} \mathfrak{I}_{k}=\mathfrak{G}_{\psi}^{\alpha, \beta}
$$

(see (5.1) and (5.3)). We have

$$
\begin{aligned}
\iint_{\mathbb{R}_{+}^{2}}(\mathcal{R} \varphi)(x, y) \overline{k(x, y)} d x d y & =\iint_{\mathbb{R}_{+}^{2}}(\mathcal{R} \varphi)(x, y) \overline{\psi\left(x^{\alpha}+y^{\beta}\right)} d x d y \\
& =\frac{2 A(\alpha, \beta)}{\alpha \beta} \int_{0}^{\infty} r^{\frac{1}{\alpha}+\frac{1}{\beta}-1} \varphi(r) \overline{\psi(r)} d r .
\end{aligned}
$$

It follows that $\mathcal{T}_{\alpha, \beta}=\mathcal{R}^{*}$ if we identify $\left(\mathfrak{B}_{p^{\prime}}^{\frac{1}{p^{\prime}}+\frac{1}{2 \alpha}+\frac{1}{2 \beta}-1}\right)^{*}$ with $\mathfrak{B}_{p}^{\frac{1}{p}+\frac{1}{2 \alpha}+\frac{1}{2 \beta}-1}$ with respect to the pairing

$$
\langle f, g\rangle=\int_{\mathbb{R}_{+}} t^{\frac{1}{\alpha}+\frac{1}{\beta}-1} f(r) \overline{g(r)} d r
$$

(see $\S 2$ ). Hence, $\mathcal{T}_{\alpha, \beta}$ is a bounded.

Suppose now that $p=1$. Clearly, (5.4) means that $0<\alpha, \beta<1$. Consider the operator

$$
\mathcal{R}: \mathfrak{b}_{\infty}^{\frac{1}{2 \alpha}+\frac{1}{2 \beta}-1} \rightarrow \mathcal{C}\left(L^{2}\left(\mathbb{R}_{+}\right)\right)
$$

defined by (5.5). It is bounded by Theorem A. Again, it is easy to see from (5.6) that $\mathcal{T}_{\alpha, \beta}^{*}=\mathcal{R}$ if we identify $\left(\mathfrak{b}_{\infty}^{\frac{1}{2 \alpha}+\frac{1}{2 \beta}-1}\right)^{*}$ with $\mathfrak{B}_{1}^{\frac{1}{2 \alpha}+\frac{1}{2 \beta}}$ with respect to the pairing (5.7).

Now suppose that $\alpha, \beta \in(0,1)$. By Theorem 5.2, the averaging projection $\mathcal{Q}_{\alpha, \beta}$ is bounded on $\boldsymbol{S}_{1}$. We can consider the conjugate operator $\mathcal{Q}_{\alpha, \beta}^{*}$ on the space $\mathcal{B}\left(L^{2}\left(\mathbb{R}_{+}\right)\right.$) with respect to the standard pairing between $\boldsymbol{S}_{1}$ and $\mathcal{B}\left(L^{2}\left(\mathbb{R}_{+}\right)\right.$) (see $\S 2$ ). Since $\mathcal{Q}_{\alpha, \beta}$ is a self-adjoint operator on $\boldsymbol{S}_{2}$, it follows that $\mathcal{Q}_{\alpha, \beta}^{*} T=\mathcal{Q}_{\alpha, \beta} T$ for $T \in \boldsymbol{S}_{2}$. We can now extend the averaging projection $\mathcal{Q}_{\alpha, \beta}$ to the space $\mathcal{B}\left(L^{2}\left(\mathbb{R}_{+}\right)\right)$ by

$$
\mathcal{Q}_{\alpha, \beta} T=\mathcal{Q}_{\alpha, \beta}^{*} T, \quad T \in \mathcal{B}\left(L^{2}\left(\mathbb{R}_{+}\right)\right) .
$$

It is easy to show that if $T$ is a bounded integral operator on $L^{2}\left(\mathbb{R}_{+}\right)$with kernel function $k$, then $\mathcal{Q}_{\alpha, \beta} T$ can be defined as in (5.1) and (5.2). 
Theorem 5.3. Let $\alpha, \beta \in(0,1)$. Then $\mathcal{Q}_{\alpha, \beta}$ is a bounded operator on the space $\mathcal{B}\left(L^{2}\left(\mathbb{R}_{+}\right)\right)$of bounded operators and on the space $\mathcal{C}\left(L^{2}\left(\mathbb{R}_{+}\right)\right)$of compact operators.

Proof. We have already explained the fact that $\mathcal{Q}_{\alpha, \beta}$ is bounded on $\mathcal{B}\left(L^{2}\left(\mathbb{R}_{+}\right)\right)$. The boundedness of $\mathcal{Q}_{\alpha, \beta}$ on $\mathcal{C}\left(L^{2}\left(\mathbb{R}_{+}\right)\right)$follows immediately from the fact that $\mathcal{Q}_{\alpha, \beta} \boldsymbol{S}_{2} \subset \boldsymbol{S}_{2}$ and the fact that $\boldsymbol{S}_{2}$ is dense in $\mathcal{C}\left(L^{2}\left(\mathbb{R}_{+}\right)\right)$.

Let us prove now that Theorems 5.2 and 5.3 are sharp.

Theorem 5.4. Suppose that $\alpha$ and $\beta$ are positive numbers such that the averaging projection $\mathcal{Q}_{\alpha, \beta}$ is bounded on $\boldsymbol{S}_{p}, 0<p<\infty$. Then $p \geq 1$ and (5.4) holds.

Theorem 5.5. Suppose that $\alpha$ and $\beta$ are positive numbers such that the averaging projection $\mathcal{Q}_{\alpha, \beta}$ is bounded on the space $\mathcal{B}\left(L^{2}\left(\mathbb{R}_{+}\right)\right)$or on the space $\mathcal{C}\left(L^{2}\left(\mathbb{R}_{+}\right)\right)$. then $\alpha, \beta<1$.

In fact for $p<1$ the following much stronger retsult holds.

Theorem 5.6. Let $0<p<1$ and $\alpha, \beta>0$. Then there is no bounded projection from $\boldsymbol{S}_{p}$ onto the subspace of operators of the form $\mathfrak{G}_{\varphi}^{\alpha, \beta}$.

Let us first prove Theorem 5.6.

Proof of Theorem 5.6. The result follows from Theorem 4.2 and the Kalton theorem [K], which says, in particular, that if $X$ is a coplemented subspace of $\boldsymbol{S}_{p}$, $0<p<1$, such that $X$ can be imbedded isomorphically to an $L^{p}$ space, then the $\boldsymbol{S}_{p}$ quasinorm and the $\boldsymbol{S}_{1}$ norm on $X$ are equivalent. Indeed, let $X$ be the subspace of $\boldsymbol{S}_{p}$ of operators of the form $\mathfrak{G}_{\varphi}^{\alpha, \beta}$. By Theorem 4.2, the $\boldsymbol{S}_{1}$ norm and the $\boldsymbol{S}_{p}$ quasinorm on $X$ are not equivalent. It follows easily from the definition of the spaces $\mathfrak{B}_{p}^{s}$ given in $\S 2$ that $X$ can be imbedded isometrically in an $L^{p}$ space. Thus by the Kalton theorem $X$ is not a complemented subspace of $\boldsymbol{S}_{p}$.

Proof of Theorem 5.4. Suppose that $1 \leq p<\infty$. The reasoning given in the proof of Theorem 5.2 shows that if the averaging projection $\mathcal{Q}_{\alpha, \beta}$ is bounded on $\boldsymbol{S}_{p}$, then the condition $\varphi \in \mathfrak{B}_{p}^{\frac{1}{2 \alpha}+\frac{1}{2 \beta}+\frac{1}{p}-1}$ implies $\mathfrak{G}_{\varphi}^{\alpha, \beta} \in \boldsymbol{S}_{p}$. The result now follows from (4.2) and from Theorem 3.1.

Proof of Theorem 5.5. If $\mathcal{Q}_{\alpha, \beta}$ is bounded on $\mathcal{B}\left(L^{2}\left(\mathbb{R}_{+}\right)\right)$or on $\mathcal{C}\left(L^{2}\left(\mathbb{R}_{+}\right)\right)$, then, by duality, $\mathcal{Q}_{\alpha, \beta}$ is bounded on $\boldsymbol{S}_{1}$. The result follows now from Theorem 5.4 .

\section{REFERENCES}

[AJPR] A.B. Aleksandrov, S. Janson, V.V. Peller, and R. Rochberg, An interesting class of operators with unusual Schatten-von Neumann behavior. In: Function Spaces, Interpolation Theory and Related Topics, Proceedings of the International Conference in Honour of Jaak Peetre on his 65th Birthday, 61-149, Walter de Gruyter, Berlin, 2002. 
[BS] M.S. Birman and M.Z. Solomyak, Spectral theory of self-adjoint operators in Hilbert space, Reidel Publishing Company, Dordrecht, 1986.

[CR] R.R. CoIfman and R. RochBerg, Representation theorems for holomorphic and harmonic functions in $L^{p}$, representation theorems for Hardy spaces, Astérisque 77 (1980), $11-66$.

[GK] I.C. Gohberg AND M.G. Krein, Introduction to the theory of linear nonselfadjoint operators in Hilbert space, Nauka, Moscow, 1965; English transl.: Amer. Math. Soc., Providence, RI, 1969.

[JP] S. Janson and J. Peetre, Paracommutators - boundedness and Schatten-von Neumann classes, Trans. Amer. Math. Soc. 305 (1988), 467-504.

[K] N.J. Kalton, Plurisubharmonic functions on quasi-Banach spaces, Studia Math. 84 (1986), no. 3, 297-324.

[Pee] J. Peetre, New thoughts on Besov spaces, Duke Univ. Press., Durham, NC, 1976.

[Pel1] V.V. Peller, Hankel operators of class $\mathfrak{S}_{p}$ and applications (rational approximation, Gaussian processes, the majorization problem for operators), Mat Sb. 113 (1980), 538-581. English transl.: Math. USSR-Sb. 41 (1982), 443-479.

[Pel2] V.V. Peller, Vectorial Hankel operators and related operators of the Schatten-von Neumann class $\mathbf{S}_{p}$, Int. Equat. Oper. Theory 5 (1982), 244-272.

[Pel3] V.V. Peller, A description of Hankel operators of class $\mathfrak{S}_{p}$ for $p>0$, investigation of the rate of rational approximation and other applications, Mat. Sb. 122 (1983), 481-510. English transl.: Math. USSR-Sb. 50 (1985), 465-494.

[Pel4] V.V. Peller, Hankel operators and their applications, to appear in Springer-Verlag.

[R] R. Rochberg, Trace ideal criteria for Hankel operators and commutators, Indiana Univ. Math. J. 31 (1982), 913-925.

[S] S. Semmes, Trace ideal criteria for Hankel operators and applications to Besov spaces, Integral Equations and Operator Theory 7 (1984), 241-281.

A.B. Aleksandrov

St-Petersburg Branch

Steklov Institute of Mathematics

Fontanka 27, 191011 St-Petersburg

Russia
V.V. Peller

Department of Mathematics

Michigan State University

East Lansing, Michigan 66506

USA 